\title{
Comparative Studies of the Structural and Transport Properties of Molten Salt FLiNaK using Machine-learned Neural Network and Reparametrized Classical Forcefields
}

Supporting Information

Shao-Chun Lee, ${ }^{\dagger, \ddagger}$ Yanqin Zhai, ${ }^{\dagger, \ddagger}$ Zhixia Li, ${ }^{\dagger, \ddagger}$ Nathan P. Walter, ${ }^{\dagger, \ddagger}$ Melissa Rose, ${ }^{\S}$ Brent J. Heuser, ${ }^{\dagger}$ and $\mathrm{Y} \mathrm{Z}^{\dagger,+, l l, *}$

tDepartment of Nuclear, Plasma, and Radiological Engineering, University of Illinois at Urbana-Champaign, Urbana, IL 61801, USA

${ }^{\ddagger}$ Beckman Institute for Advanced Science and Technology, University of Illinois at Urbana-Champaign, Urbana, IL 61801, USA

$\S$ Division of Chemical and Fuel Cycle Technologies, Argonne National Laboratory, 9700 S. Cass Avenue, Lemont, IL 60439, USA

"Department of Electrical and Computer Engineering, University of Illinois at Urbana-Champaign, Urbana, IL 61801, USA

Email: zhyang@illinois.edu 


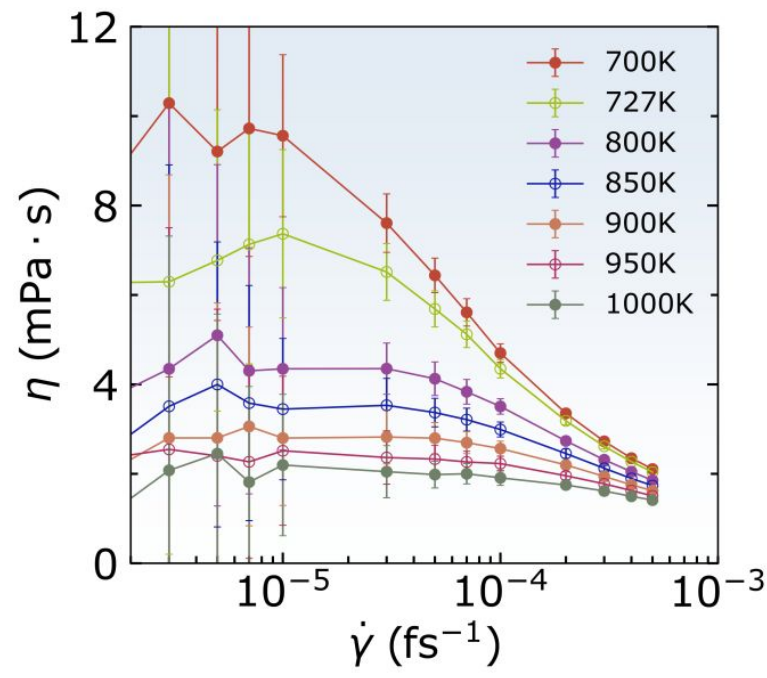

Figure S1. Viscosities computed with NEMD method as a function of strain rate and temperature.
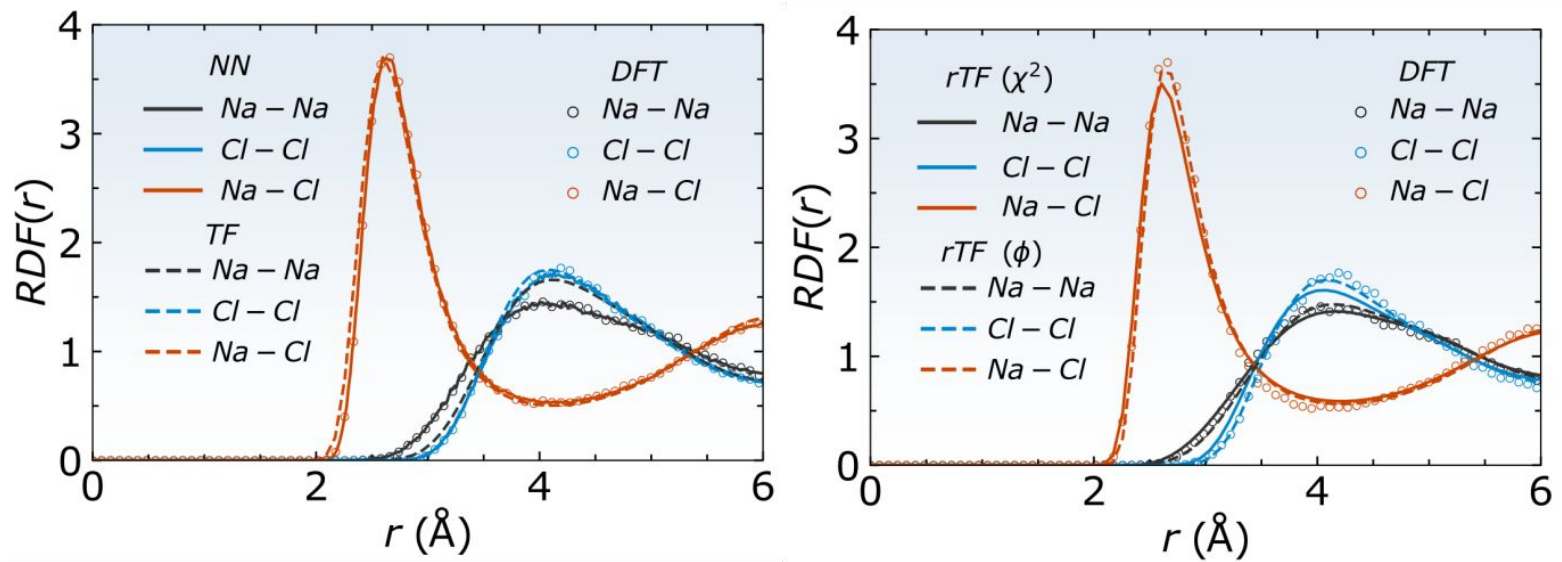

Figure S2. RDFs of molten NaCl at 1273 K. (A) Comparison between NN (solid), TF (dashed), and DFT (scatter). (B) Comparison between rTF fitted with $\chi^{2}$ (solid), $\phi$ (dashed), and DFT (scatter)
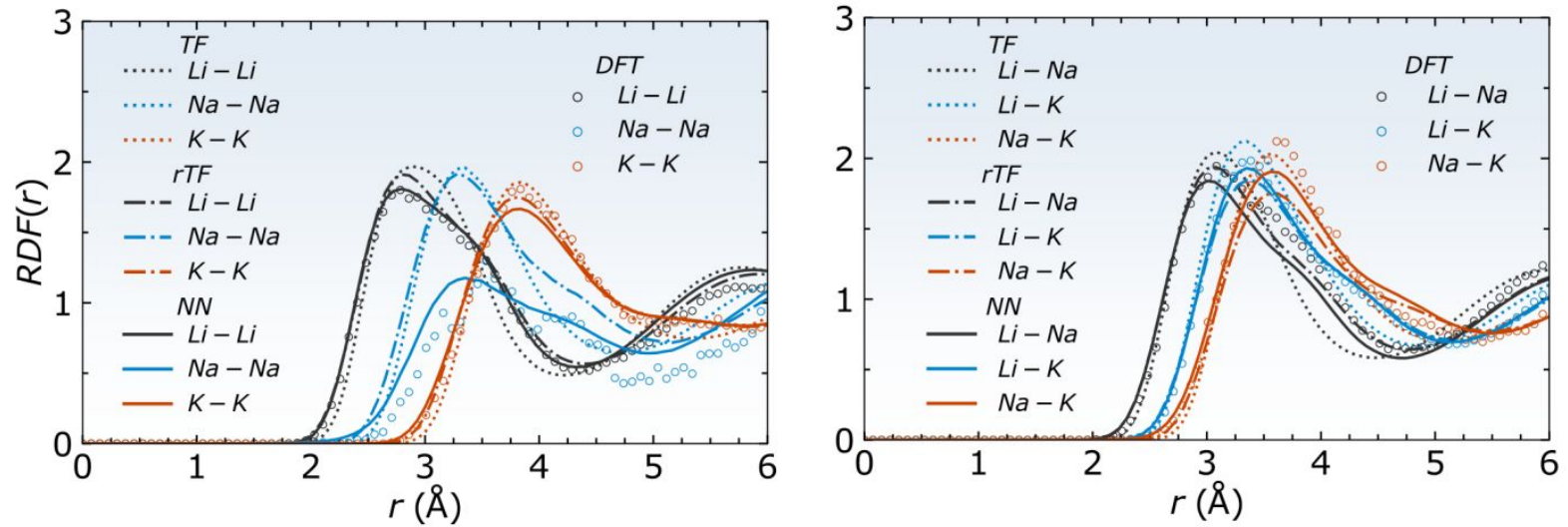

Figure S3. RDFs of cation-cation pairs for FLiNaK at 1000 K. (A) Like-pairs. (B) Unlike-pairs. 


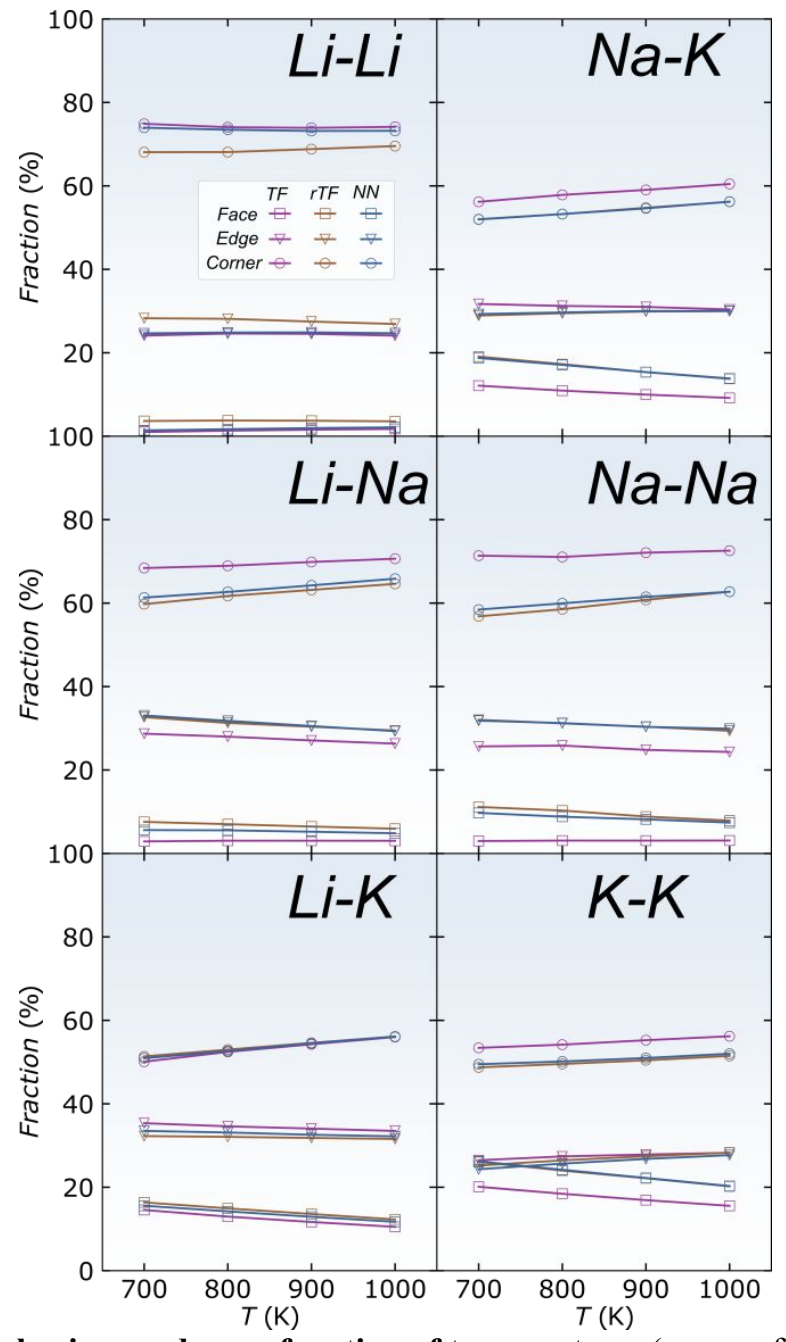

Figure S4. Fraction of $\mathbf{F}$ ion sharing mode as a function of temperature. (square: face sharing, triangle: edge sharing, circle: corner sharing, purple: TF, yellow: rTF, NN: blue)
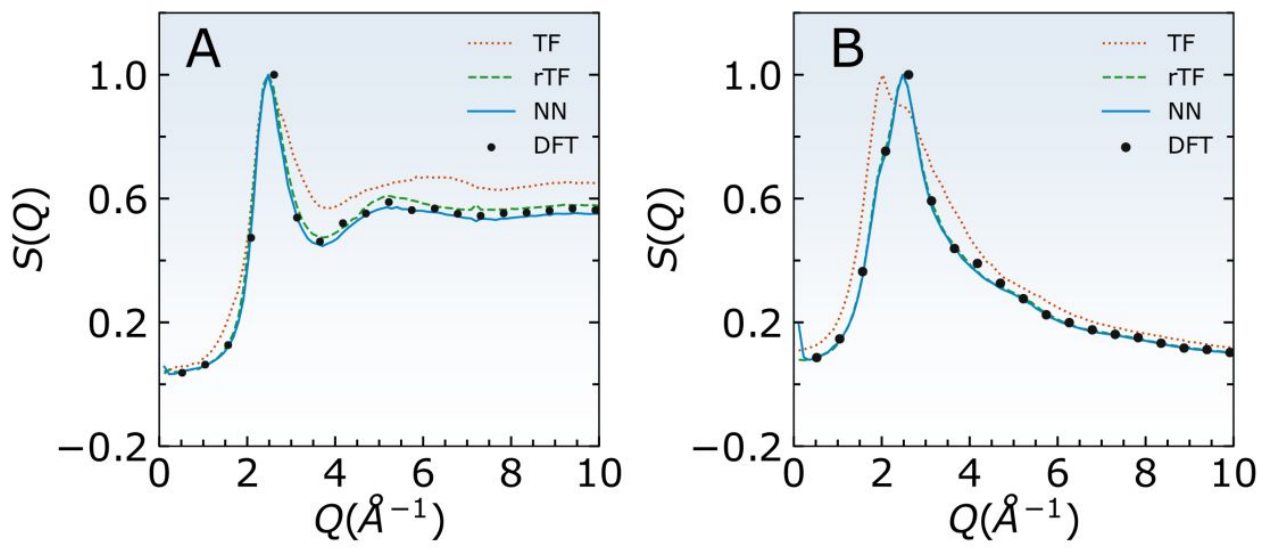

Figure S5. Structure factors for FLiNaK at 1000 K. (A) Weighted by neutron scattering lengths (B) Weighted by $\mathrm{x}$-ray atomic form factors 
Table S1. Parameters of RIM for molten NaCl.

\begin{tabular}{|c|c|c|c|c|c|c|}
\hline $\mathrm{i}-\mathrm{j}$ & $\mathrm{C}_{\mathrm{ij}} \mathrm{b}(\mathrm{kcal} / \mathrm{mol})$ & $\sigma_{\mathrm{ij}}(\AA)$ & $\rho_{\mathrm{ij}}(\AA)$ & $\mathrm{C}_{\mathrm{ij}}\left(\mathrm{kcal} / \mathrm{mol} \cdot \AA^{6}\right)$ & $\mathrm{D}_{\mathrm{ij}}\left(\mathrm{kcal} / \mathrm{mol} \cdot \AA^{8}\right)$ & $\mathrm{q}_{\mathrm{i}}$ \\
\hline \multicolumn{7}{|c|}{$\mathrm{TF}$} \\
\hline $\mathrm{Na}-\mathrm{Na}$ & 6.079 & 2.340 & 0.317 & 24.170 & 11.510 & 1.000 \\
\hline $\mathrm{Cl}-\mathrm{Cl}$ & 3.647 & 3.170 & 0.317 & 1669.020 & 3352.440 & -1.000 \\
\hline $\mathrm{Na}-\mathrm{Cl}$ & 4.863 & 2.755 & 0.317 & 161.150 & 200.000 & \\
\hline \multicolumn{7}{|c|}{$\mathrm{rTF}\left(\chi^{2}\right)$} \\
\hline $\mathrm{Na}-\mathrm{Na}$ & 6.828 & $2.340^{\mathrm{a}}$ & 0.143 & 15.537 & 13.205 & 0.722 \\
\hline $\mathrm{Cl}-\mathrm{Cl}$ & 4.375 & $3.170^{\mathrm{a}}$ & 0.338 & 1571.323 & 3094.825 & -0.722 \\
\hline $\mathrm{Na}-\mathrm{Cl}$ & 1.814 & $2.755^{\mathrm{a}}$ & 0.232 & 171.129 & 190.612 & \\
\hline \multicolumn{7}{|c|}{$\mathrm{rTF}(\phi)$} \\
\hline $\mathrm{Na}-\mathrm{Na}$ & 3.146 & $2.340^{\mathrm{a}}$ & 0.367 & 18.979 & 6.610 & 0.775 \\
\hline $\mathrm{Cl}-\mathrm{Cl}$ & 5.809 & $3.170^{\mathrm{a}}$ & 0.350 & 1653.794 & 3353.921 & -0.775 \\
\hline $\mathrm{Na}-\mathrm{Cl}$ & 2.125 & $2.755^{\mathrm{a}}$ & 0.229 & 151.618 & 193.226 & \\
\hline
\end{tabular}

${ }^{a}$ Fixed to original TF values

Table S2. Parameters of RIM for molten FLiNaK.

\begin{tabular}{|c|c|c|c|c|c|c|}
\hline $\mathrm{i}-\mathrm{j}$ & $\mathrm{c}_{\mathrm{ij}} \mathrm{b}(\mathrm{kcal} / \mathrm{mol})$ & $\sigma_{\mathrm{ij}}(\AA)$ & $\rho_{\mathrm{ij}}(\AA)$ & $\mathrm{C}_{\mathrm{ij}}\left(\mathrm{kcal} / \mathrm{mol}^{\circ} \AA^{6}\right)$ & $\mathrm{D}_{\mathrm{ij}}\left(\mathrm{kcal} / \mathrm{mol} \cdot \AA^{8}\right)$ & $\mathrm{q}_{\mathrm{i}}$ \\
\hline \multicolumn{7}{|c|}{$\mathrm{TF}$} \\
\hline $\mathrm{F}-\mathrm{F}$ & 3.647 & $2.358^{\mathrm{a}}$ & 0.302 & $236.714^{\mathrm{a}}$ & $279.778^{\mathrm{a}}$ & -1.000 \\
\hline $\mathrm{Li}-\mathrm{Li}$ & 6.079 & 1.632 & 0.299 & 1.050 & 0.430 & 1.000 \\
\hline $\mathrm{Na}-\mathrm{Na}$ & 6.079 & 2.340 & 0.330 & 24.170 & 11.510 & 1.000 \\
\hline $\mathrm{K}-\mathrm{K}$ & 6.079 & 2.926 & 0.338 & 349.630 & 345.320 & 1.000 \\
\hline $\mathrm{F}-\mathrm{Li}$ & 4.863 & $2.047^{\mathrm{b}}$ & $0.271^{\mathrm{b}}$ & $0^{\mathrm{b}}$ & $0^{\mathrm{b}}$ & \\
\hline $\mathrm{F}-\mathrm{Na}$ & 4.863 & 2.349 & 0.330 & 64.750 & 54.670 & \\
\hline $\mathrm{F}-\mathrm{K}$ & 4.863 & 2.642 & 0.338 & 280.570 & 302.150 & \\
\hline $\mathrm{Li}-\mathrm{Na}$ & 6.079 & 1.986 & $0.281^{\mathrm{c}}$ & $5.36^{\mathrm{c}}$ & $2.63^{\mathrm{c}}$ & \\
\hline $\mathrm{Li}-\mathrm{K}$ & 6.079 & 2.279 & $0.299^{\mathrm{c}}$ & $166.48^{\mathrm{c}}$ & $164.11^{\mathrm{c}}$ & \\
\hline $\mathrm{Na}-\mathrm{K}$ & 6.079 & 2.633 & $0.336^{\mathrm{c}}$ & $279.67^{\mathrm{c}}$ & $273.56^{\mathrm{c}}$ & \\
\hline \multicolumn{7}{|c|}{$\mathrm{rTF}(\phi)^{\mathrm{d}}$} \\
\hline $\mathrm{F}-\mathrm{F}$ & 4.978 & 2.340 & $0.238^{2}$ & 207.209 & -328.306 & -0.817 \\
\hline $\mathrm{Li}-\mathrm{Li}$ & 6.853 & 1.429 & 0.212 & 0.996 & -0.406 & 0.794 \\
\hline $\mathrm{Na}-\mathrm{Na}$ & 4.534 & 2.156 & 0.260 & 22.990 & -9.172 & 0.829 \\
\hline $\mathrm{K}-\mathrm{K}$ & 7.290 & 2.726 & 0.311 & 380.490 & -319.882 & 0.840 \\
\hline
\end{tabular}

${ }^{a}$ Weighted average from Li-F, Na-F and K-F pair by mole $\%$.

b Suggested in the literature ${ }^{25}$.

${ }^{\mathrm{c}}$ Weighted average from corresponding ion pairs by mole $\%$.

${ }^{\mathrm{d}}$ Arithmetic mixing was applied for $\sigma$; geometric mixing rules are applied for all other parameters.

Table S3. The $\chi_{F}^{2}$ and $\chi_{E}^{2}$ values averaged over 20 selected DFT snapshots by different models for molten $\mathrm{NaCl}$. Parentheses after $\mathrm{rTF}$ indicate the loss function used in the reparameterization.

\begin{tabular}{|l|c|c|}
\hline Model & $\chi_{\mathrm{F}}^{2}$ & $\chi_{\mathrm{E}}^{2}$ \\
\hline TF & $0.94 \pm 4.26$ & $0.03 \pm 0.001$ \\
\hline rTF $\left(\chi^{2}\right)$ & $0.13 \pm 0.45$ & $0.14 \pm 0.001$ \\
\hline rTF $(\phi)$ & $0.15 \pm 0.58$ & $0.08 \pm 0.001$ \\
\hline NN & $0.03 \pm 0.096$ & $0.002 \pm 0.0003$ \\
\hline
\end{tabular}


Table S4. Average CNs evaluated by different forcefields for each cation.

\begin{tabular}{|c|c|c|c|}
\hline Model & $\mathrm{Li}^{+}-\mathrm{F}^{-}$ & $\mathrm{Na}^{+}-\mathrm{F}^{-}$ & $\mathrm{K}^{+}-\mathrm{F}^{-}$ \\
\hline TF & 3.87 & 4.57 & 7.38 \\
\hline rTF & 4.12 & 5.25 & 7.78 \\
\hline NN & 3.90 & 5.02 & 7.64 \\
\hline DFT & 3.93 & 4.96 & 7.64 \\
\hline
\end{tabular}

Table S5. Computational efficiency tested on the 12-core single node with Intel Xeon CPU E5-2670 v2.

\begin{tabular}{|c|c|c|c|}
\hline Method & DFT & TF/rTF & NN \\
\hline Code & VASP & LAMMPS & LAMMPS \\
\hline System Size & 100 atoms & 10000 atoms & 10000 atoms \\
\hline Computational speed & 0.0014 timestep/s & 37.7 timestep $/ \mathrm{s}$ & 3.0 timestep/s \\
\hline
\end{tabular}

